\title{
A SURVEY ON GREEN CLOUD COMPUTING
}

\author{
Jai A. Mehta, Pankti K.Nanavati, Vishant K. Mehta \\ Department of IT \\ KJSCE, Mumbai, Maharashtra, India
}

\begin{abstract}
Green cloud computing is about designing cloud computing resources in a manner which reduces its impact on the surrounding environment. Green clouds can save a lot of energy and are also capable of reducing operational costs. This is a necessity today as global warming levels continue to rise across the planet. As more and more businesses shift towards cloud, it is important for all cloud providers to adopt green cloud computing. This paper provides an overview of green cloud computing and also talks about the ways in which it can be achieved. It also talks about the applications and challenges being faced for the implementation of green clouds.
\end{abstract}

Keywords-Cloud computing, Green cloud computing, Environment Sustainability, Energy Consumption.

\section{INTRODUCTION}

Cloud Computing is the use of various computer system resources like storage, computing power (without the use of any external resources by the user), etc. over the internet. With the rising computational demand and data storage in today's world, cloud computing has developed into a wide research field. As a result of these technical advances, the next move should be to create environmentally sustainable, energy-efficient, and cost-effective solutions. IT services use a lot of power and energy. Global climate changes, such as increased $\mathrm{CO} 2$ emissions and energy shortages, could result as a result of this. As a consequence, "Green Cloud Computing," which is capable of generating explications that are both cost- effective and energy-efficient IT resources, is a must. Using computers and other devices more effectively would result in increased resource usage, energy efficient peripherals, and reduced electronic waste. Green Computing is the name given to this definition. Green computing can improve resource efficiency as well as performance.

\section{NEED FOR GREEN CLOUD COMPUTING}

Today in the modern world, our lives cannot be imagined without the use of any kind of technology. Due to the use of a lot of technology based products, we are also having to deal with a large amount of data. This huge amount of data is stored in various data centers across the whole world. These data centers need a lot of energy and power to be able to run smoothly and efficiently all the time and this is also a necessity because you would never want the server to go down. Almost all the fields in the modern age are dependent on Information Technology. Healthcare, Banking, Media, Automobiles, etc all use IT on a regular basis and their work would almost stop without it right now. There are many other fields where Information Technology is used on a vast scale which has led to increasing energy consumption over the years. Eventually, which leads to increasing costs.

There have also been many advancements in the field of Technology in the recent years. Among which, one of them is Cloud Computing which is in very high demand right now. Basically, it means that we can use various computing services without having to actually use any extra hardware on our side. Everything happens remotely over the internet and we get to use the computational power of a remote server on our local machine. Cloud Computing has changed the way we use technology now. It has proved to be of massive help in various fields. But the thing is, with the increase in the use of Cloud Computing we are also having to deal with a ton of data which is even more than before now. And as most of the data centers have started using the Cloud Computing architecture now, the amount of Carbon emissions have also increased which can cause serious damage to the environment. We cannot even restrict the use of Cloud Computing now because it has become a very important part of our lives, but what we can do is to develop ways to reduce these harmful emissions.

Then, there's IoT (Internet of Things) which deals with physical products that use sensors and softwares inside them to connect and communicate over the Internet. Whenever we hear terms like "connecting", "data", "internet", etc in the same sentence we should pretty much figure that all these things are possible due to Cloud Computing and large data centers present at the location of the remote servers. IoT thus, is also dependent on data centers for most of its infrastructure. And data centers as it is logical consume a lot of energy. The energy consumption in fact is on a steady rise due to the innovation and emergence of new technologies and products.

The field of Health Care Medicine also makes extensive use of technology and also the use of Cloud Computing has increased over the years in the field. These things have revolutionized the way the healthcare sector works. We can see the use of equipment powered by the latest technology, improved equipment for surgical work, being able to consult doctors remotely over the internet, online healthcare platforms and many other things. This has become possible 


\section{International Journal of Engineering Applied Sciences and Technology, 2021}

Vol. 6, Issue 1, ISSN No. 2455-2143, Pages 425-429

Published Online May 2021 in IJEAST (http://www.ijeast.com)

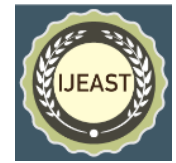

due to Technology and Cloud Computing. But at the same time, the healthcare field also faces challenges regarding how to make energy efficient and safe use of such tools and techniques. A study also shows that Healthcare also plays a major role in the carbon footprint of countries like the USA, Australia, etc. The energy use of the healthcare field is estimated to grow even more in the coming years with the emergence of new technologies.

So, we need to find a way to reduce the amount of energy consumed while still being able to utilize these resources. This has made way for Green Cloud Computing which basically means the use of Cloud Computing in an environment friendly manner. Green Cloud Computing tries to provide eco-friendly and economically viable use of computing resources while still providing the same value to the users. Many companies worldwide have also started investing in the developments of Green Cloud Computing. These are all the reasons which have made Green Cloud Computing necessary in today's world.

\section{APPROACHES}

Green cloud computing provides multiple solutions to alle- viate the impact of cloud computing on the environment. There are various different approaches and techniques proposed for achieving green cloud computing. There are mainly three ways: first is the hardware optimization which includes reduc- ing the use of energy and making it economically efficient, second is software optimization which includes developing ways to increase efficiency of energy, storage and program, and the last one is network optimization.

\section{A. Virtualization}

It is a very common approach used in green cloud computing. There are different types of virtualization of which server virtualization plays a major role because servers play the most important role in a Cloud Data centre. In this approach multiple VMs (Virtual Machines) are assigned to a single server. This can be done by the use of a software application. It helps is reducing the cost to a great deal of hardware and operating costs, consolidation of tasks and reduction in energy use (power consumption) by switching off physical devices. Virtualization also allows to move a running virtual machine from one host to another with no downtime and distributed power management possible.

\section{B. Green Scheduler}

The green scheduler or the green scheduling algorithm determines which servers should be turned on and turned off.

When the load increases, the server will be turned on and when the load decreased the server will be turned off automatically. Since, servers take time to load completely, they should be switched on before it is needed. The servers should also not be loaded more than its capacity. This leads to reduction in energy and power consumption at the data centre. It also helps in reducing the load on the servers at any instant of time since at any given point of time there are already requisite number of servers which are on.

\section{Datacentre Energy Efficient Network Aware Scheduling Algorithm}

This algorithm helps in reducing the cost and operational expenses of data centres by minimizing the total energy consumption. It selects the best fit resources for executing a particular task or problem on the basis of the load as well as taking into consideration the various components present at the data centre. Also, for managing the workload, a deadline- based model is employed which aims at completion of each task within a specified amount of time. Hence, it achieves workload efficiency by preventing the components(servers) from overloading and network congestion. However, there is a small amount of increase in the number of servers that are running.

\section{Nano Data Centres}

It is a computing platform which is distributed. They refer to the large number of data centres with smaller sizes than the normal data centres which are large in sizes and lesser in number. The creation of nano data centres helps in reducing the energy consumption by 30 percent. They are distributed around the world and are interconnected. They are portable and can be used anywhere including remote locations or for temporary use. They help in the reduction of downtime with a decrease in response time.

\section{E. Use of Tranquil PCs}

There is a large amount of carbon emissions due to all the data centres using cloud computing architecture. The use of tranquil PCs causes the carbon emission to reduce to approximately $60 \mathrm{~kg}$ per year as opposed to the desktop PCs in the datacentres which consume approximately $400 \mathrm{KW}$ of power and even with the power saving option approximately $270 \mathrm{~kg}$ of Carbon dioxide is produced per year. Hence, the use of tranquil PCs helps in reducing the carbon emissions.

\begin{tabular}{lrrr}
\hline Parameters & $\begin{array}{l}\text { Per } \\
\text { week }\end{array}$ & $\begin{array}{l}\text { Per } \\
\text { month }\end{array}$ & $\begin{array}{l}\text { Per } \\
\text { year }\end{array}$ \\
\hline $\begin{array}{l}\text { Reference desktop Pc's } \\
\text { Costs }\end{array}$ & 6.9 & 29.4 & 35.19 \\
CO2 Emissions & 4.3 & 18.1 & 2.18 \\
$\begin{array}{l}\text { Tranquil Pc's } \\
\text { Costs }\end{array}$ & 2.2 & 9.4 & 111.6 \\
\hline CO2 Emissions & 1.9 & 5.7 & 67.90 \\
\hline
\end{tabular}

F. ASDI Methodology 


\section{International Journal of Engineering Applied Sciences and Technology, 2021}

Vol. 6, Issue 1, ISSN No. 2455-2143, Pages 425-429

Published Online May 2021 in IJEAST (http://www.ijeast.com)

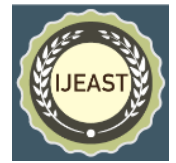

ASDI stands for Analysis Specifications Design Implemen- tation, which can be used for designing and implementation of a model. It helps in decomposition of a system into three main subsystems- first is the PSS i.e., the Physical Subsystem, second LSS i.e., the Logical Subsystem and the third DSS i.e., Decision making Subsystem. It provides more efficient and adoptable modelling approach for green cloud computing data centres. This methodology makes decision making pro- cess hierarchical, easy facilitation of decision-making process, distribution of the power of resolution tools and cooperation of decision entities. Another advantage is that ASDI methodology is easily understandable by anyone, even those who are no specialist. It aims at improving the design of green cloud computing in collaboration with engineers.

\section{G. TOE model}

TOE refers to Technical Organization Environment. It accounts for both technical as well as non-technological aspects such as the environment and organizational factors. It comprises of technical, organizational and environmental factors. The technical factor refers to technical side such as implementation and design of cloud data centres, the organizational factor refers to the organization of policies and protocols for ensuring green cloud computing along with the knowledge of the latest and new green cloud computing equipment. The environmental factor includes instructing the cloud providers on standards and policies of green computing and sustainability. The TOE model aims at increasing the efficiency and also reducing the carbon footprint due to the use of technology.

\section{H. Dynamic Migration Algorithm}

The main objective of the algorithm is to reduce the power and energy consumption as well as reduce carbon dioxide emissions. It helps in increasing the efficiency of resource utilisation. But the drawback is that it exceeds migration costs and takes longer than usual to respond i.e. longer response time.

\section{Using ARtificial Intelligence to ACHIEVE GREEN CLOUD COMPUTING}

As green cloud computing mainly revolves around reducing the energy consumption at data centers, artificial intelligence can help achieve this goal by means of intelligent resource scheduling and intelligent refrigeration. Due to unprecedented variations in requests from the cloud users, resource scheduling is an important factor in reducing the energy consumption. Optimal usage of resources is important when the demand surges. If resources are not allotted optimally, a large amount of energy gets consumed unnecessarily. Traditional approaches do not use AI for resource allocation, and though these strategies perform decently while allocating resources, these can be further optimized by usage of AI.

\section{A. Scheduling control engine}

When a request is sent, the optimization scheduling module collects data related to all the resources present in the data center with the help of a resource sensing layer. Using AI, resource allocation is done based on the current request submitted by the user and the current state of the resources.

- Resource perception module: Deep learning predicts the future load and in tandem with the current load, the scheduling control engine does the resource allocation. Since the future load is predicted in tandem with the current load, resource allocation can be optimized. As more and more data is trained, the deep learning model gets better at predictions of the future load. Deep learning models thrive on a large amount of data.

- Optimization scheduling module: Based on inputs from the resource perception module this module can reserve resources while allocation of resources takes place. Resource allocation was based on time required for completing the job by using algorithms like FCFS or based on priority. Reinforced learning can be used to learn each time a scheduling takes place.

- Policy verification module: Scheduling policy thus formed by the optimization and scheduling module needs to be verified by the policy verification module. This is mainly done for safety reasons so that the constraints of the system are not violated and the data center can operate smoothly. Also, it may happen that the scheduling model is currently weak as not enough data has been fed into the model. In such cases, the policy verification module scraps the schedule generated by the model itself and uses traditional approaches like FCFS. The scheduling policy makes sure that the resources which are no longerneeded, can be switched off.

- Self-learning module: Self-learning module is used to enhance the knowledge base of the system.

\section{B. Intelligent refrigerating engine}

When the resources are used for computation, heat is produced in the nodes, which needs to be dissipated as soon as possible. This is mainly because the physical equipment in the room may find itself harmed if the heat is not dissipated quickly. Refrigeration thus becomes a necessity. Intelligent refrigerating engine uses deep learning to predict energy consumption in data centres. Refrigeration takes up a lot of energy. Information related to the 


\section{International Journal of Engineering Applied Sciences and Technology, 2021 Vol. 6, Issue 1, ISSN No. 2455-2143, Pages 425-429 \\ Published Online May 2021 in IJEAST (http://www.ijeast.com)}

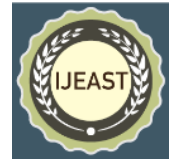

surrounding environment is taken into considerationtemperature, moisture, airflow and current status of resources is also taken into account. Model is used to check the correlation between energy consumption and environment information. Parameters associated with the refrigeration are thus determined and suitable operations are performed. The refrigeration thus has an intelligence of its own which decreases the total operational cost and also reduces the carbon footprint.

\section{Applications of Green Cloud Computing}

\section{A. Green Internet Of Things}

The Internet of Things (IoT) connects smart objects to a heterogeneous network to allow monitoring and decisionmaking. The increase in the number of IoT devices is posing a threat to cloud computing platforms. Since these IoT devices require low latency and mobility, edge computing for real- time services has emerged. Fog computing is a distributed computing model aimed at linking network devices at various levels of computation. They offer IoT devices a low-latency answer that centralised cloud computing infrastructure can't match. Green computing focuses on maintaining computing resources while reducing energy consumption and being environmentally friendly. By utilizing machine resources, Green computing allows for a more environmentally sustainable use of energy and other activities. It entails redesigning and eliminating various computing elements in order to minimize environmental damage. The aim of green computing is to use computing resources in a way that is both environmentally sustainable and cost-friendly. Green computing uses various technological domains:

- Autonomous vehicles: Autonomous vehicles must send data to their manufacturers in order for them to monitor their use and receive necessary maintenance warnings. Edge computing facilitates data transmission and sharing between autonomous vehicles. They often reduce the amount of energy used by sensors in autonomous vehicles. The risk of carbon emissions is minimized as aresult of the move toward autonomous vehicles, which is a step toward a more environmentally sustainable approach.

- Smart cities: City leaders use the data obtained from sensors, which includes traffic, infrastructure, and home appliances, to solve the problems that these cities face. The response time to these devices should be instantaneous, resulting in less energy consumption.

- Industries: Oil exploration for example, may use IoT edge computing to collect data on a range of environmental factors without relying on previously collected data. As a result of the introduction of edge computing in industries, there would be less energy use in manufacturing.

\section{B. Green Healthcare}

Virtualization of information technology (IT) data centre devices is the most important step most companies will take to move to green healthcare. While cost reduction is often the driving force behind virtualization, IT versatility is often the most important factor. The cost and energy savings from consolidating hardware and software are substantial, and they complement the usability advantages very well.

The dominant Green IT practices in healthcare are:

- Use of Electronic Medical Records (EMRs) EMR is used by healthcare professionals to track, control, and coordinate health care delivery within a healthcare organisation. EMRs have the ability to minimise carbon dioxide emissions, according to estimates. Users revealed that by using an EMR, they were able to save thousands of pounds of paper for medical records each year. As a result, the environment had a net positive impact.

- Telemedicine: Telemedicine is the practice of medicine that uses technologies to give treatment to people who are located far away. Telemedicine has been around for more than two decades, but its effects are only now becoming apparent, especially in rural areas. It helps to reduce carbon consumption as people can avoid going all over for expert referrals and other events. It can be used to help treat chronic conditions, optimise treatment for the sick, homebound, and physically challenged, and boost community and population wellbeing.

\section{Green Parallel Computing of Big Data Systems}

Big Data is usually structured around a distributed file system on top of which parallel algorithms for Big Data analytics can be run. The parallel algorithms can be mapped to the computing platform in a number of ways. In terms of environmentally related parameters such as energy and power usage, each choice would behave differently. Current research on the implementation of parallel computing algorithms have largely focused on addressing general computing metrics such as speedup over serial computing and efficiency of the use of computing nodes. We explore how to elicit green metrics for big data systems, which are necessary when comparing implementation options. We use current systematic literature reviews to define and address the key green computing indicators for big data systems.

\section{CHALLENGES IN GREEN COMPUTING IMPLEMENTATION}

\section{A. Green Computing Awareness}

There exists a general lack of understanding about green cloud computing among the people. People are generally unaware about its impact on the environment. People need 
to be convinced how important it is to have an infrastructure supporting green computing. Awareness among public needs to be raised. Otherwise, the adoption of green computing is a distant dream.

\section{B. Equipment Cost}

People feel that it is better to use traditional approaches because those are not very costly, but such a thought process is likely to backfire for humanity in the longer run. People need to be cautious about the impact their products are having on the environment either directly or indirectly.

\section{CONClusion}

As more and more businesses are switching to cloud, the amount of energy utilized by these cloud data centers is increasing at a rapid rate and is significantly contributing towards carbon footprint. Green cloud computing provides a solution to this problem by reducing energy consumption and optimizing resource allocation. Powerful AI techniques are aiding the growth of green cloud computing. Green computing can be implemented in various fields like IoT and big data analytics. Public needs to be educated about the importance of green computing. Adopting green computing in the future will be extremely beneficial for the environment.

\section{REFERENCES}

[ 1 ] Manoj Muniswamaiah, Tilak Agerwala and Charles C. Tappert, "Green computing for Internet of Things", 2020 7th IEEE International Conference on Cyber Security and Cloud Computing (CSCloud).

[ 2 ] J.M.T.I. Jayalath, E.J.A.P.C. Chathumali, K.R.M. Kotha- lawala, N. Kuruwitaarachchi, "Green Cloud Computing: A Review on Adoption of Green-Computing attributes and Vendor Specific Implementations", 2019.
[ 3 ] Mridul Wadhwa, Approv Goel, Tanupriya Choudhury, Ved P Mishra, "Green Cloud Computing - A Greener Approach To IT", 2019 International Conference on Computational Intelligence and Knowledge Economy (ICCIKE).

[4] Jun Yang, Wenjing Xiao, Chun Jiang, M. Shamim Hossain, Ghulam Muhammad, Syed Umar Amin, "AIPowered Green Cloud and Data Center", 2018 IEEE Access.

[5] Nina S. Godbole, John Lamb, "Research into Making Healthcare Green with Cloud, Green IT, and Data Science to Reduce Healthcare Costs and Combat Climate Change", 2018 IEEE.

[6] Surendran.R, Tamilvizhi.T, "How to Improve the Re- source Utilization in Cloud Data Center?", 2018 International Conference on Innovation and Intelligence for Informatics, Computing, and Technologies (3ICT).

[7] Fatima Shakeel, Seema Sharma, "Green Cloud Computing: A review on Efficiency of Data Centres and Virtualization of Servers", 2017 International Conference on Computing, Communication and Automation (ICCCA).

[ 8 ] Mr. Nitin S. More,Dr. Rajesh B. Ingle, "Challenges in Green Computing for Energy Saving Techniques", 2017 International Conference on Emerging Trends Innovation in ICT (ICEI).

[9] Havva G u"lay Gürbu"z, Bedir Tekinerdogan, "Software

Metrics for Green Parallel Computing of Big Data Systems", 2016 IEEE International Congress on Big Data.

[ 10] Jean-Charles Huet, Ikram El Abbassi, "Green Cloud Computing modelling methodology", 2013 IEEE/ACM 6th International Conference on Utility and Cloud Computing. 